\title{
A Smart Optimization of Fault Diagnosis in Electrical Grid using Distributed Software-Defined IoT System
}

\author{
Ammar K. Al Mhdawi, Member, IEEE, Hamed Al-Raweshidy, Senior Member, IEEE
}

\begin{abstract}
Electrical power demands have increased significantly over the last years due to the rapid increase in air conditioning units and home appliances per domestic unit particularly in Iraq. Having uninterrupted power supply is essential for the continuity of power-generated home services and industrial platforms. Currently, in Iraq, electrical power interruption has become a big concern to the utility suppliers even with the successive attempts in putting end to this dilemma, but the issue still prevailed. One of the main factors in power outages in local zones is persistent faults in distribution transformers (DT). DT is considered one of the main elements in the electrical network that is essential for the reliability of the grid supply. Due to the internal lack of monitoring system and periodic maintenance, DT is relentlessly subject to faults due to high overhead utilization. Therefore, in order to enhance the grid reliability, transformer health check and maintenance practices, we propose a remote condition IoT monitoring and fault prediction system that is based on a customized Software-Defined Networking (SDN) technology. This approach is a transition to smart grid implementation by fusing the power grid with efficient and real-time wireless communication architecture. The SDN implementation is considered in two phases; one is a controller installed per local zone and the main controller that is installed between zones and connected to the core network. The core network consists of redundant links to recover from any future fails. Furthermore, we propose a prediction system that is based on Artificial Neural Network algorithm called Distribution Transformer Fault Prediction (DTFP) that is installed in the management plane for periodic prediction based on real-time sensor traffic to our proposed cloud. Moreover, we propose a communication protocol in the local zone called Local SDNsense. The SDN-sense ensures a reliable communication and local node selection to relay DT sensor data to the main controller. Our proposed architecture showcase an efficient approach to handle future interruption and faults in power grid using costeffective and reliable infrastructure that can predict and provide real-time health monitoring indices for the Iraqi grid network with minimal power interruptions. After extensive testing, the prediction accuracy was about $96.1 \%$. The
\end{abstract}

Index Terms-Software-Defined Networks (SDN), neural networks (NN), smart grid, monitoring network, fault prediction, LoRa-IoT, sensors.

\section{INTRODUCTION}

Generally, high power has to be generated and supplied to the domestic and industrial units on a 24/7 basis. The power source and distribution network of the electrical system has to be maintained continuously to provide non-stop electricity consumption. Traditional power grid relies on human operators to manage and monitor the status and the efficiency of the grid and coordinate supply and demands to ensure reliable stability of the power grid [1]. The significant increasing requirements for quality power management is implemented via deploying monitoring and control strategies all over the grid system. Traditional distribution transformers have an average life of 20-25 years; however, most of these transformers are at the end cycle of their life and are posing an intermittent risk to the power grid system. The current monitoring system of the power grid in Iraq is only associated with major electrical parameters that provide no health check status on the internal components of the local distribution network. Lack of Periodic maintenance and follow up checks is a major factor in these repetitive DT failures that is due to non-established visibility system. Therefore, a robust monitoring and prediction system is needed to establish real-time monitoring of each distribution unit of the local grid [2] by using SDN principle. Software-Defined Networking or (SDN) is a new programmable network concept paradigm that has been proposed recently to facilitate management and data steering of the network. SDN is the concept of separating the control plane from the data plane in which the forwarding hardware is segregated from the decision-making platform such as routing and control software [3]. The separation of the planes provides a flexible, programmable and cost-effective network infrastructure. In the SDN network, the policies will be running on the controller only instead of running them on each device as in the traditional network. The controller will have a full overview of the network topology and all nodes can be configured from single point of management. This approach will provide a robust management of large scale network with less overhead. Each engine has a table called forwarding table that forwards on the basis of matching the incoming packet to the table. The communication between the SDN and OpenFlow switches is governed by the OpenFlow protocol. The OpenFlow protocol is a set of messages that are exchanged between the controller and the switches over a secure connected channel. The controller sends modification messages to the switch node such as add, modify, remove entries from the forwarding table. When an incoming packet enters the OpenFlow switch, it maps the packet info to the forwarding table, if there is a match, then it forwards the packet to the designated port; otherwise, it sends a query request to the controller to request advice from the controller on where to send the packet. The SDN controller then consults its topology table and decide whether to send new rules or notify the switch to drop the packet. Furthermore, SDN has two main interfaces, one is Northbound Interface (NBI) that is used to push configuration, read, install rules and implement 
modifications on excising rules. The second interface is the Southbound Interface or (SBI) that is used by the controller to push rules and modification to the lower level nodes or OpenFlow switches. [4]-[8].

To implement a structural health monitoring system for the distribution transformers, a wireless sensor network (WSN) is considered. A WSN is a network that is constructed using a large number of distributed nodes where each node consists of a specific sensor that detects a physical condition of an object such as temperature, heat, liquid levels, pressure, etc. Sensor nodes monitor the condition and transmit the data along to other nodes until it reaches the management node or gateway that represents the collection of all data. The sensors are powered by either a fixed power source such as batteries or by using an energy harvesting technology such as solar, thermal or kinetic [9]. However, wireless sensors are limited by over the air transmission obstacles that could hinder the transmission rate of data comparable to the wired network systems. Installation of sensors on electrical grid components can provide an immediate status of components condition which helps in understanding how the grid can handle a certain electrical load and can provide early fault alert with minimum low cost of repair. The result of using WSN correlate with the increase in profitability and stability of the electrical grid .

Traditional tools are not always capable of achieving efficient accuracy and reliability regarding fault classification of distribution transformers. The process of identifying faults in the DT components is significantly crucial for the continuity of the power supply. It can help in reducing the number of unexpected faults, reduce maintenance cost and help in extending the life cycle of the transformer [10]. Additionally, by using a smart intelligent system, it becomes a coherent process to assist in analysis and fault classification of the operational transformer based on its current load status. Moreover, Neural Networks (NN) has been greatly used in the electrical power network for predicting power production and estimating power demands.

Recently, researchers have been using statistical modeling and methods to evaluate and analyze the behavior of the power grid network. However, NN is considered a new approach in prediction compared to conventional prediction methods. The strength of NN is that they do not need any assumptions and they use previous historical data to generate prediction by optimizing the non-linearity issues in the system. The prediction is done by constructing a complex relationship between the input and the output by applying rounds of training and optimization on a given dataset [11]. Moreover, $\mathrm{NN}$ consist of neurons or perceptrons that are interconnected with each other via links. There are three main layers in a neural network that are the input layer, hidden layer, and an output layer. A perceptron has multiple inputs to it with weights for each link. Details of the proposed neural networks architecture are described later in Section III, respectively.

\section{RELATED WORK}

Software-defined networks or SDN [12] have played a significant role in reconstructing the network architecture to less complex and flexible elements in terms of deployment and flexibility. Moreover, Neural Networks (NN) has been establishing a solid ground in many sectors by predicting the status of system behavior and provide accurate predictions based on historical data. Nonetheless, researchers have been working on different modeling techniques to implement the neural network in power grid to predict power supply performance and fault diagnosis. Below, we list some of the work that was implemented by researchers to put solutions for some of the challenges and concerns that occurred in the power grid as follows:

Grid component faults are significant problems in power distribution, for that, Senlin et al. [13] proposed a method for prediction of the trip fault using long-short-term- memory and support vector machines which are a high margin classifier in neural networks. The data were captured with the LSTM network with a long time span. About 500 sampling of voltage, current and active power was collected during normal operation. The data were fed into the proposed system and result in $97 \%$ accuracy rate in trip fault prediction. Hengxu et al. [14] presented a novel solution for distribution feeder relays for predicting the faults levels. This technique implemented with two main inputs voltage and current of the breakers. The fault current was calculated using Thevenin's theorem and actual measurement was compared. The output of the neural network algorithm showed an accuracy of about $98 \%$ with less than $2 \%$ error rate. Moreover, Yuan et al. [15] proposed a systematic approach that investigates the fault of power electronics elements under different working conditions. Investors and rectifiers are crucial elements in power conversion. However, the life cycle of these components is influenced by a concurrent number of operations. The author implemented multiple machine learning techniques that have taken into account the operation condition and data imbalance for efficient converters fail prediction. multiple probabilistic models have been used such as SVM and SOM. The final results showed variance with the best prediction for the ensemble classification.

Mohammed et al. [16] proposed an artificial system for predicting the power network stability after the fault is cleared. The input variables were used are fault statues such as prefault, during-fault, and post-fault valuers. The proposed neural network uses the cross-entropy function as the cost function to optimize the weights, and the softmax is used as the activation function. data were divided into three sets, $60 \%$ for training, $20 \%$ for validation and finally $20 \%$ for testing. The results of the simulation have shown an overall accuracy of $99.3 \%$. Fei et al. [17] presented a statistical neural network approach for predicting the power quality disturbances that may affect the power grid. The author has used the multi-hidden Markov model. The data set of power quality disturbance and weather condition were used as the main data to train the model. Moreover, the author has used Hadoop clustering to process the data efficiently and to reduce computation time. The author provided that an improvement of $20 \%$ were achieved compared to other model used. Younghun et al. [18] proposed a predictive neural network model to predict and evaluate the dissolved gas analysis in substation transformers based on the previous history of operation. Optimization technique has been 
used to solve the fitting issue. The data were collected from seven substation transformers. Transformer's health status is recorded using the SCADA monitoring system. standard mean absolute error and percentage have been used for the regression performance check. After extensive testing, the prediction error of each dissolved gas generated by increased oil temperature in the transformer index is very low that are $15 \%$ for $\mathrm{H}_{2}$, $7 \%$ for $\mathrm{C}_{2} \mathrm{H}_{2}, 5 \%$ for $\mathrm{C}_{2} \mathrm{H}_{4}, 5 \%$ for $\mathrm{C}_{2} \mathrm{H}_{6}$ and $1.5 \%$ for $\mathrm{CH}_{4}$. The prediction error is limited within $2 \%$ for each gas level prediction. The overall prediction accuracy is between $84 \%$ to $97 \%$. Huang et al. [19] presented a monitoring system for large scale IoT in countryside areas. The author have deployed 19 LoRa nodes over area with dimensions of $800 \mathrm{~m} \times 600 \mathrm{~m}$ with access gateway of $1 \mathrm{~min}$ interval data collection. The author provided that the PDR ratio for the proposed mesh network achieved about $88.49 \%$ while traditional star topology achieved $58.7 \%$. The author have added that the project aim is to explorer the potential of IoT mesh deployment architecture in areas that require long range transmission. Zefang et al. [20] proposed optimization clustering method for mixed data for SDN-based smart grid networks. The output algorithm is based on a a combination of k-means and k-modes algorithms. The author provided that the proposed algorithm satisfies the differential privacy experiment with efficient accuracy. Kun $e t$ al. [21] adopted an energy efficient sense layers architecture to address the energy that is consumed by large number of IoT nodes. The author provided that the proposed framework is in three layers, that are sense, gateway and control layers. The author used sleep and wake scheduling protocol with prediction of sleep intervals. Furthermore, the author has deployed in simulation 300 nodes in a large area, whereas 250 nodes are for sensing and 50 as gateways. After extensive testing, the results shows that a significant drop in power consumption improving resource utilization and energy consumption. Kun et al. [22] discussed concerns of dense deployment of small cells that are inconsistent interfaces, frequent handovers and extensive backhauling. The author have introduced SDN for the NWNs architecture by decomposing the control plane from the data plane. The author have used virtual RATs design to support different services. The author concluded that the proposed SDNC is able to predict user's movement path that is near the AP to implement the handover. After extensive testing, the author added that the proposed approach was validated and handover is thus accelerated and overall latency is reduced. Kun et al. [23] The author discussed the large amount of data that is generated from big data platforms such as health monitoring networks that require real-time processing and analysis. Many of these data is not needed and cause delay in processing and storage. Therefore, the author proposed an RVNS optimization search method that operate in three layers. For that reason, the author have used three layers approach that are fault-tolerant approach to ensure the reliability of the eHEALTH system and second is the layer that checks for accuracy of the data and the final layer is where RVNS optimization is implemented where only valuable data will be reported to the health provider system for processing. This approach help efficiently increase processing time and delivery ratio. Min et al. [24] proposed multiple approaches starting with a probabilistic modelling using Markov Chain method to verify the energy routing system in smart green city networks and MDP model to check the cost of the service requester and provider. The author also introduced a monitoring tool over the ER system to monitor the scheduling process. The processing of power transactions process were implemented in the cloudlet platform.

The main contributions of this paper can be summarized as follows:

- We propose a customized SDN infrastructure that consists of long-range power IoT sensor network called Grid Management Network (GMN). The GMN consist of two parts: (1) the wireless sensor network section that is implemented on each distribution transformer per each zone. A list of sensors is installed on each transformer such as a temperature sensor, oil level sensor, humming noise sensor and over-loading sensor. These sensors represent the health status check for each distribution transformer. Each one of these sensors is linked to an RF transmitter using long-range LoRa WAN communication. The second part of the architecture is (2) wireless-static data center. The data center consist of multiple paths to provide redundancy with fault tolerance route recovery. The network will use SDN controller as the gateway entry node to the static data center.

- We propose a Fault Prediction algorithm (DTFP) for fault prediction in distribution transformer. The neural network algorithm consists of multiple interconnected mesh hidden layers with various weights. Optimization is implemented using back probation (BP) to tune the weights for efficient prediction accuracy. The DTFP is installed in the management layer with periodic fault prediction based on hourly historical data.

- We propose a communication protocol called Software Defined communications (SDN-sense) for the wireless IoT nodes on the distribution transformers network. The protocol runs on both layers, control layer represented as sink node and forwarding layer representing the forwarding engines. The forwarding tables are built using received $\mathrm{BC}$ packets and then information is relayed to the sink SDN node for constructing the topology table. The best route is selected to be used as the main route; however, an alternative fail-recovery is addressed with the most reliable route.

The remainder of the manuscript is organized as follows. In Section III, system testbed architecture is presented. Section IV, experimental results and analysis are discussed and explained. Finally, section VI is the conclusion were a summarization of the work is illustrated.

\section{SYSTEM TESTBED ARCHITECTURE}

Our proposed grid management network consists of realvirtualized hardware components that run on a Linux server. The core network data center runs on pure sdn architecture that consists of two SDN controllers with a fail-over capability and forwarding engines as OpenFlow vswitch (2.9.2) [25]. The operating system platform we have used is the Ubuntu 


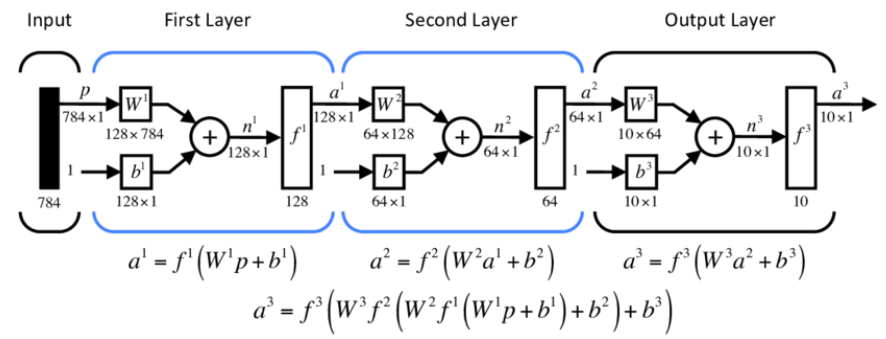

Fig. 1: multi-layer perceptron architecture diagram [29]
The main factor in calculating the error level in our prediction model and to test the usefulness of our fault prediction platform is the Mean Square Error (MSE) and Root Mean Square Error(RMSE), which both typically are called objective or cost function. The cost has to be a very small value in order for our system to be reliable in fault prediction analysis. The MSE and RMSE can be expressed as in eq 1 and eq 2. The difference between the two equations is that taking the RMSE gives high weights to large errors which can be used exceptionally useful when undesirable errors occur.

$$
\begin{gathered}
\operatorname{Obj}\left(\mathrm{x}_{1}, x_{2}, \ldots, x_{n}\right)=\frac{1}{n} \sum_{i=1}^{n}\left(F l t_{\text {pred }}-F l t_{\text {trgt }}\right)^{2} \\
\operatorname{Obj}\left(\mathrm{x}_{1}, x_{2}, \ldots ., x_{n}\right)=\sqrt{\frac{1}{n} \sum_{i=1}^{n}\left(F l t_{\text {pred }}-F l t_{\text {trgt }}\right)^{2}}
\end{gathered}
$$

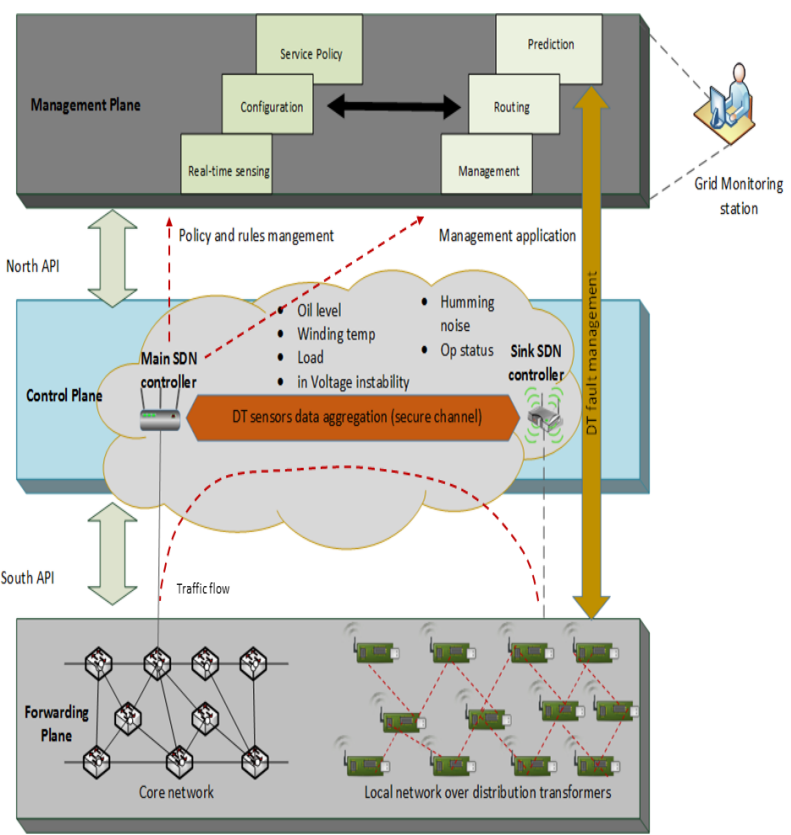

Fig. 2: proposed architecture block diagram

The block diagram of the proposed architecture is depicted in Fig 2. The diagram consists of a forwarding plane that represents the OpenFlow engines for the core and wireless network. The next layer is the control plane that represents the data steering and route management platforms. A secure channel is established between the main sdn and sink sdn controller for data security and reliability. Furthermore, the top layer is the management layer that represents the storage and real-time sensing platform with the fault prediction network that is trained repeatedly with new events every hour. The re-training is implemented using the backpropagation model. After using our developed proposed Distribution Transformer Fault Prediction (DTFP) algorithm as in Fig 3, we found that the proposed model

In order to track the overall status of the DT system, We assume a status index (SI) factor of the distribution 


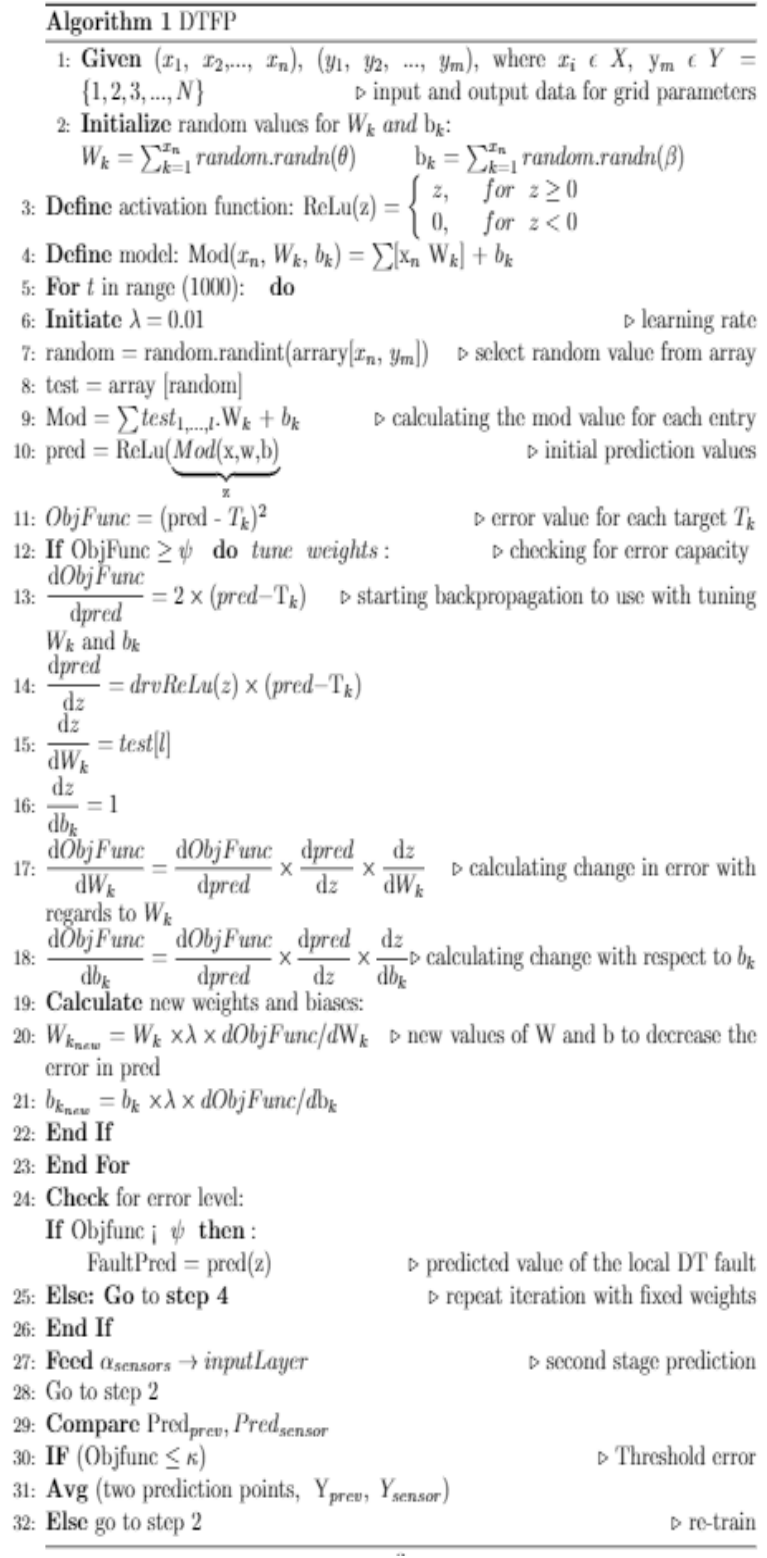

Fig. 3: proposed DTFP algorithm pseudo code

$$
S I(\%)=\frac{1}{1+e^{-} \sum_{i=1}^{n}\left(\alpha x_{i}\right)} \times 100
$$

where $\alpha$ represents the weight effect of each sensor variable that ranges between (1-10). We can classify the SI index of the DT health status as follows in Table I:

TABLE I: Condition Status Index

\begin{tabular}{ll}
\hline Status Index (\%) & Condition \\
\hline \hline $100<\mathrm{SI}<90$ & very good \\
\hline $80<\mathrm{SI}<70$ & good \\
\hline $70<\mathrm{SI}<65$ & yellow alert (require investigation) \\
\hline $\mathrm{SI}<60$ & system critical (fail) \\
\hline
\end{tabular}

The proposed communication algorithm between the IoT nodes is governed using SDN-sense algorithm as described in Fig 4. The distribution transformer that is being investigated is described in Table II as follows:

TABLE II: Investigated Transformer Specification

\begin{tabular}{ll}
\hline Parameter & Description \\
\hline \hline Rated voltage(max) & $11 \mathrm{kVA}$ \\
\hline Rated voltage (low) & $433 \mathrm{v}-250 \mathrm{v}$ \\
\hline Load current (max) & $3.3 \mathrm{~A}$ \\
\hline Load current (high) & $84 \mathrm{~A}$ \\
\hline Connection & Delta \\
\hline No. of phases & 3 \\
\hline Frequency & $50 \mathrm{c} / \mathrm{s}$ \\
\hline Noise level & $50 \mathrm{db}$ \\
\hline Operating average temperature & $35-40$ Deg.C \\
\hline
\end{tabular}

IV. EXPERIMENTAL RESULTS AND ANALYSIS

The proposed complete smart grid architecture based SDN is described in Fig 5. We can notice that the core network that is represented as the cloud consist of multi-path routing links governed by SDN enabling forwarding engines to operate their designated operating requests. Furthermore, each specified zone is connected via a mesh network of SDN and OpenFlow switches that relay sensor data to the main SDN controller which is installed at the edge of the cloud. The cloud that we used in our experimental testing is based on virtualized environment represented in virtual machines. The SDN controller is implemented using mininet and floodlight controller. The rules were configured in advance and set to be installed in the Open vswitch engines.

The wireless nodes communication is based on our proposed algorithm SDN-sense is shown in Fig 4. Moreover, the architecture described in Fig 5 represents the overall proposed architecture that combines the core network represented in the cloud, the SDN wireless mesh network and the fault prediction system. We consider the SDN architecture as a directed graph $G=(S W, L)$, where $S W$ represents all the OpenFlow switches ( SDN to be in case of failure), $L$ represents a set of RF links $L=\{(i, j) \in S \times S, i \neq j\}$. The SDN and OF switches are customized and programmed to match our case study, and the SDN can be accessed via a python API for 


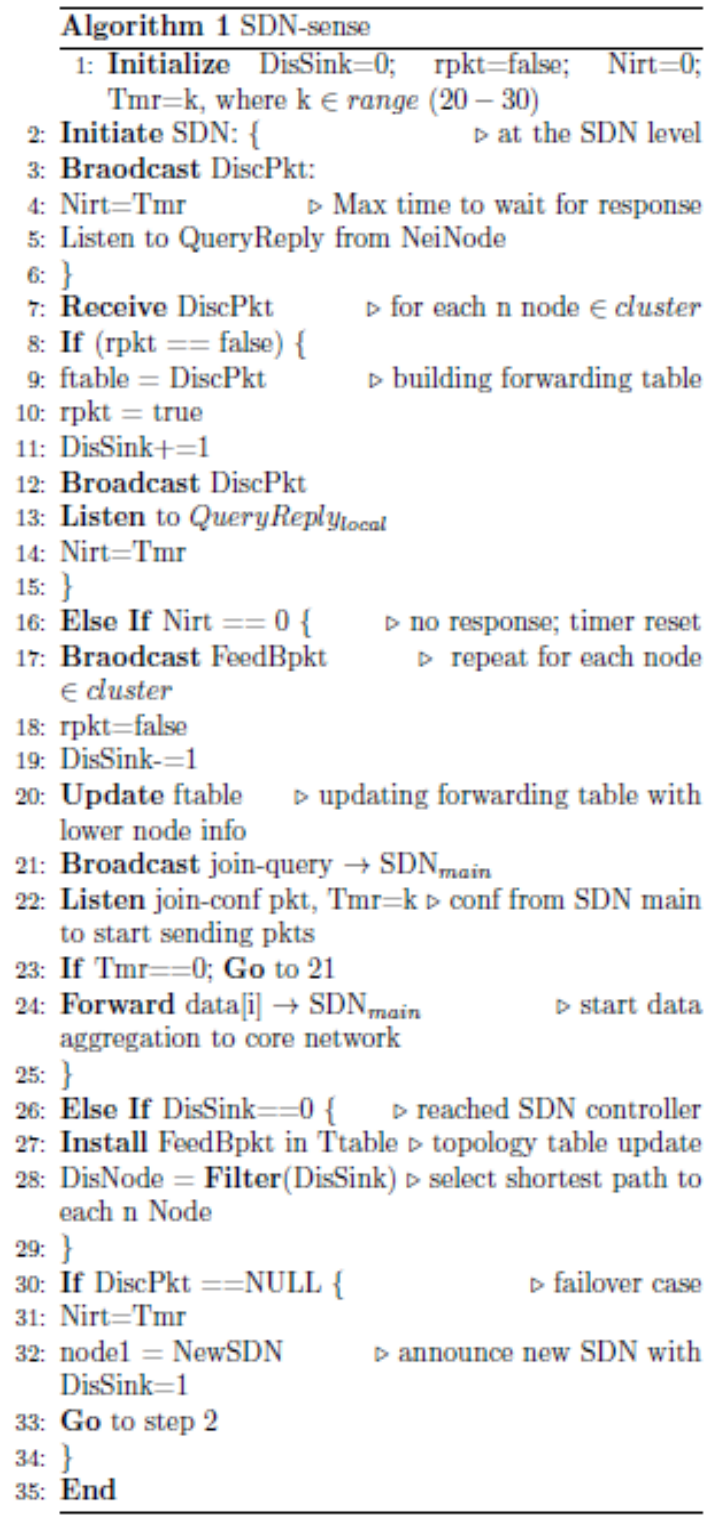

Fig. 4: proposed SDN-sense pseudo-code

further modification and data retrieval. Furthermore, the OF forwarding table can be represents as $F=\left\{\lambda_{p k t}, \beta_{t a b}, \alpha_{a c t}\right\}$, where are three main objects composes the forwarding table that are flows, tables and actions. Each packet is required to be matched to a table then a decision is made on where to forward the packet based on a bucket of actions. The number of rules that are existed in a particular OF node can be represented in Eq 4 as follows:

$$
R_{k}=\sum_{1}^{n} \Delta_{k, t}
$$

where $\Delta_{k, t}$ represents the rule per OF node with $t$ as an indication for sub-rule. $k$ is subscript of total rules. The total matching delay that may occur in the OpenFlow table can be denoted in Eq 5 as follows:

$$
\phi_{\text {match-delay }}=\sum_{1}^{N} R_{k} \times \sigma_{q-\text { factor }}
$$

where $\sigma_{q-\text { factor }}$ is the queueing delay for processing flows that can affect the total processing capacity of the OF node significantly. Although power consumption of the SDN sink node is not high, it is worthily to mention it as it may affect on the lifetime of the node sensor and designing an efficient power management node can result in efficient power consumption and longevity of the operating node. The power consumption of the SDN sink and main SDN node can be expressed in Eq 6 and Eq 7 as follows:

$$
\begin{aligned}
P_{\text {sink } k_{\text {total }}=} & \sum_{1}^{n} \theta_{\text {temp }}+\sum_{1}^{n} \theta_{\text {oil }}+\sum_{1}^{n} \theta_{\text {temp }}+ \\
& \sum_{1}^{n} \theta_{C-i n}+\sum_{1}^{n} \theta_{V-i n}+\sum_{1}^{n} \theta_{\text {lora }}
\end{aligned}
$$

where $\theta$ represents the inbound traffic power consumption of a specific sensor.

$$
\begin{array}{r}
P_{S D N_{\text {main }}}=\sum \lambda_{\text {clust }_{1}}+\sum \lambda_{\text {clust }_{2}}+\sum \lambda_{\text {clust }_{3}} \\
+. .+\sum \lambda_{\text {clust }_{n}}+\sum \lambda_{\text {lora }}+\sum \lambda_{\text {init }}
\end{array}
$$

\section{HARDWARE IMPLEMENTATION AND DEPLOYMENT}

In this section, we present the proposed sensor hardware that can be implemented in a residential transformer zone. The system is built using an IoT off-the-shelf hardware that is programmed with SDN implementation principle. The hardware unit of the sensor consists of an Arduino board that is programmed as a microcontroller board with SDN functionality. The proposed hardware consists of five main sensors that are temperature sensor, oil level sensor, humming noise sensor, AC-in sensor, and V-in sensor. The sensor nodes based on OpenFLow platform communicate with sink SDN node using a long-range communication network by implementing LoRa network due to the heterogeneity of the communication in such environment. The main gateway or SDN main responsible for managing the communication with all sink nodes and to collect all sensor data to be forwarded for aggregation to the data center. After data is processed and stored, they will be fed to the prediction system so that a fault prediction can be produced based on real-time sensor data. The prediction can help in identifying any future faults that could occur in the D-Transformer and to re-route power and isolate faulty D-transformer for a maintenance procedure. Fig 6 shows the proposed SDN IoT hardware prototype with components labeled, whereas, Fig 8 virtualized data center implementation that runs on a Linux server. The server represents the core network that uses network function virtualization (NFV) for efficient power consumption management. The prediction system runs on the servers under python library. In our IoT testbed, we have used Arduino Uno [30] and programmed it as a customized SDN sink controller that operates with a LoRa module LX1278 with a custom-tailored antenna for 


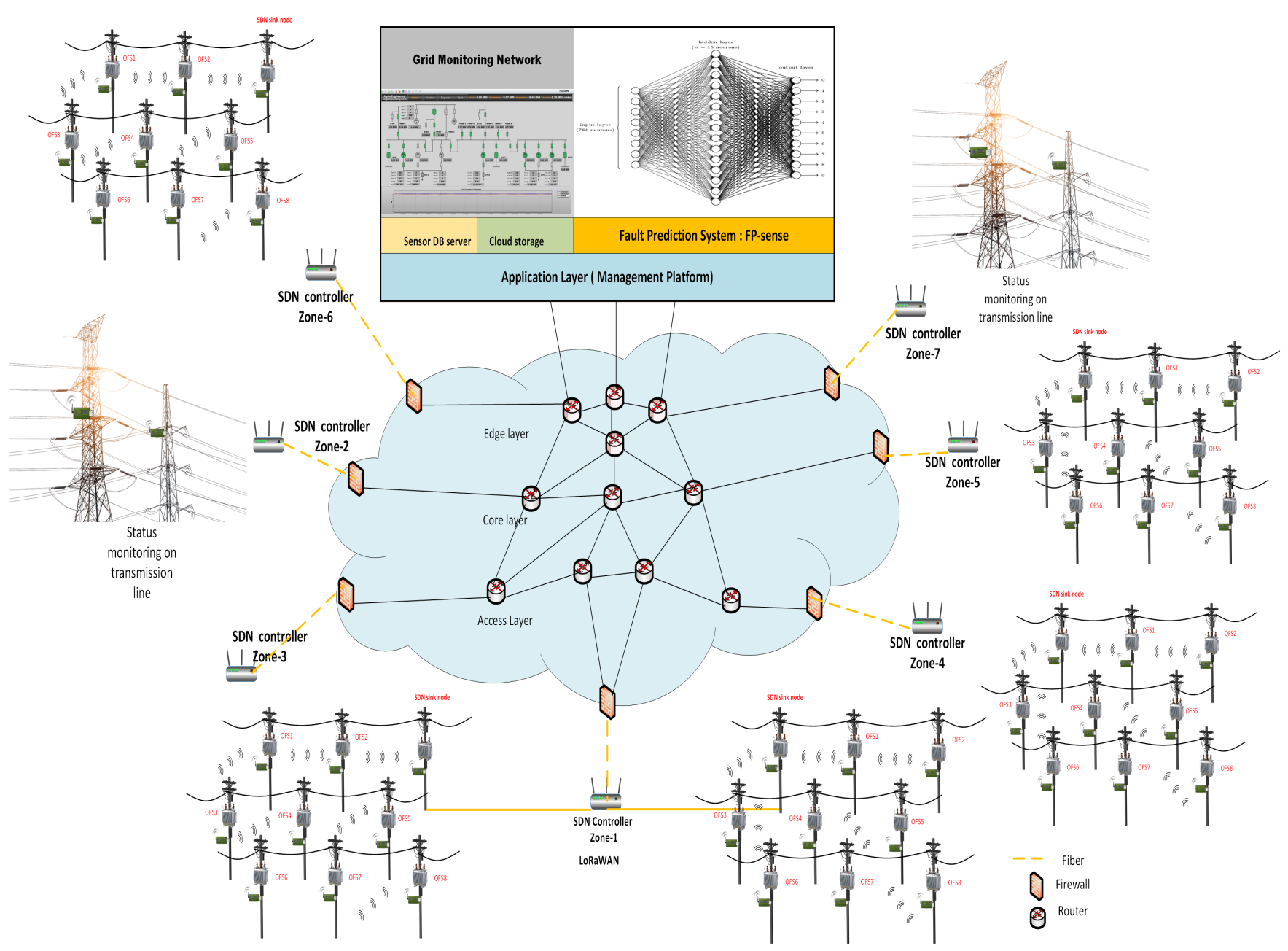

Fig. 5: Grid Management Network (GMN) proposed architecture platform

better signal gain and propagation. The rest of the sensors are AC sensor ACS712 with AC voltage sensor ZMP101B. For the temperature sensor, we have used thermo-couple sensor MAX6675 with an ultrasonic sensor to measure the humming noise HCSR04. The sink node is powered with $9 \mathrm{v}$ power supply. Fig 7 shows the final enclosure box for the proposed testbed. This box is designed for a single phase only for this current research project. The three main cables are for $\mathrm{AC}-\mathrm{V}$, $\mathrm{AC}-\mathrm{C}$ and $\mathrm{CB}$ for switching and transformer protection.

Fig 9 shows faulty transformers images that were collected from different grid sites in Iraq that were effect by many factors such as shorted winding, high temperature fault, high incoming voltage and oil leaks. Additionally, damages could be caused due pivot pole fall which causes total damage to the D-transformer outer case.

In Fig 10 above, we present a deployment case scenario of our proposed sdn sink sensor over residential transformers. The sink node communicates to the SDN master node using LoRa RF communication then to the cloud network for sensor data processing. The IoT-based sensor node is based on sensing and action implementation based on the level of incoming data from each sensor. Many sensors have been implemented in our testbed such as oil level sensor, temperature sensor, AC voltage sensor, and AC current sensor. Based on these data, an action will be made to cut off the circuit breaker in case of a high alert. Additionally, these data will be fed to the prediction system for statistical analysis based on real-time data and historical environmental data. A decision will be generated from the prediction system to regulate the transformer behavior and to reduce any future fails. The testbed prototype that we have implemented only suits for single case transformer scenario. However, it operates as a testbed that can operate with three phase system. The testing was implemented on a small miniature scale transformer due to limited resources. Fig 11 shows sensor readings for a single-phase transformer that depicts the health index of each part as shown.

In order to build our proposed prediction system, we have used historical data set for the outages and faults based on records that were logged by the grid transformer maintenance workshop in Iraq. The dataset includes data such as fault time, fault date, fault type and fault no. of occurrences. These attributes were taken as input to the neural network with an additional sensor data that can increase the accuracy in a form of double stage input. The hidden layer as we see in Fig 12 consist of 10 layers with the sigmoid as the objective function.

The weights were initialized randomly at first stage then 


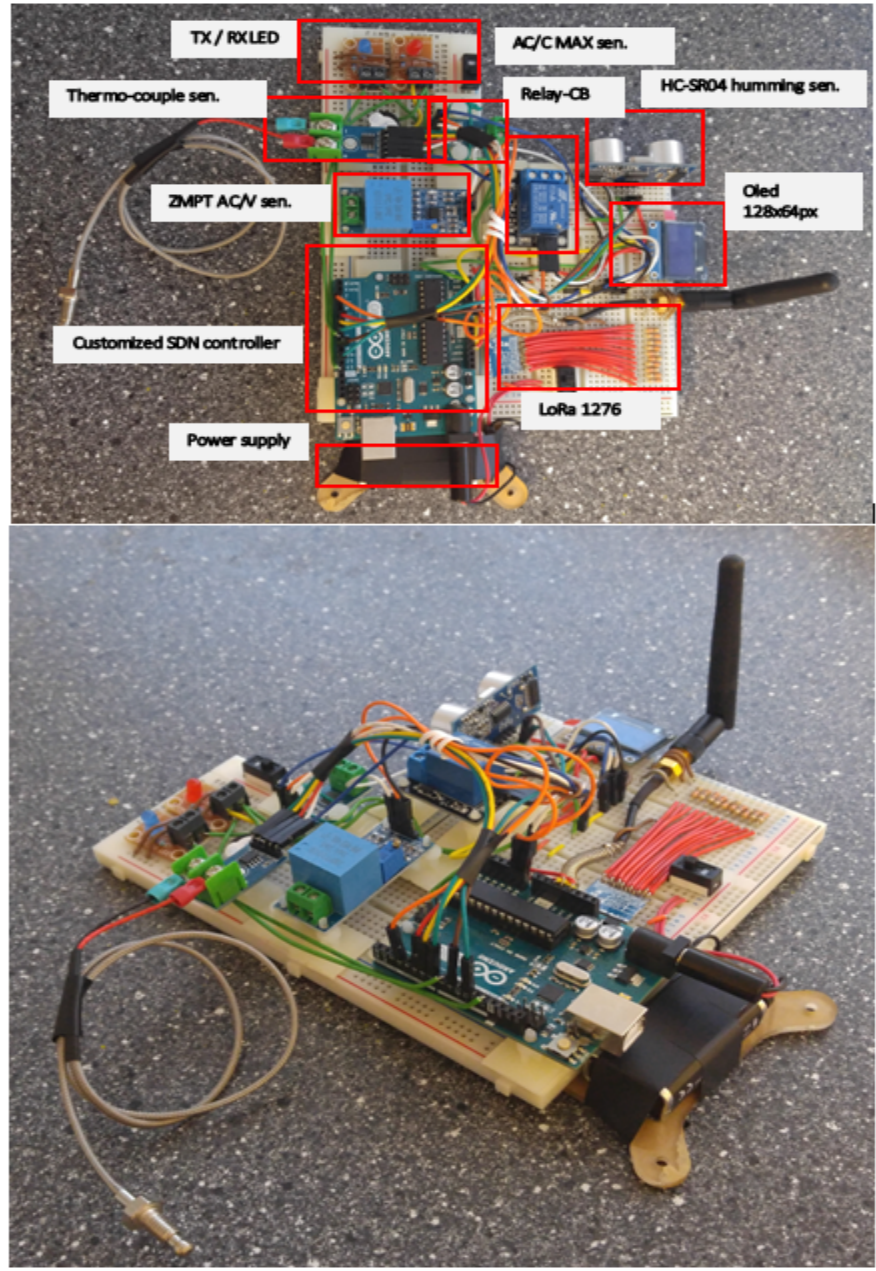

Fig. 6: proposed OpenFlow IoT sensor Platform

used backpropagation to tune the weights for better prediction accuracy. However, for final stage prediction, we have used Decision Tree classification algorithm for accurate prediction. We have implemented a combination of feed-forward for sensors and historical dataset and decision tree algorithm for finding the best average prediction of historical and real combined sensor data output. We can notice that a better accuracy has been achieved by using our proposed work of feed forwarding and decision tree averaging algorithm while minimizing the error rate between each $Y$ prediction value. Furthermore, The sensor data were fed to our proposed prediction platform and we were able to get a low error rate after 1000 rounds of training as depicted in Fig 13. The optimization of the error rate can be reduced more by using more critical relational parameters that can be estimated for each transformer.

Fig 14 represents the data set parameters that were used to train our proposed model. The main input data are the line trip, frequency, line load, and voltage. In Fig 15 shows the prediction of the type of fault and phase line overload with $96.1 \%$ accuracy. The accuracy can be optimized more by using more operational parameters. Moreover, Fig 16 shows the gradient decent process parameters that are used to tune

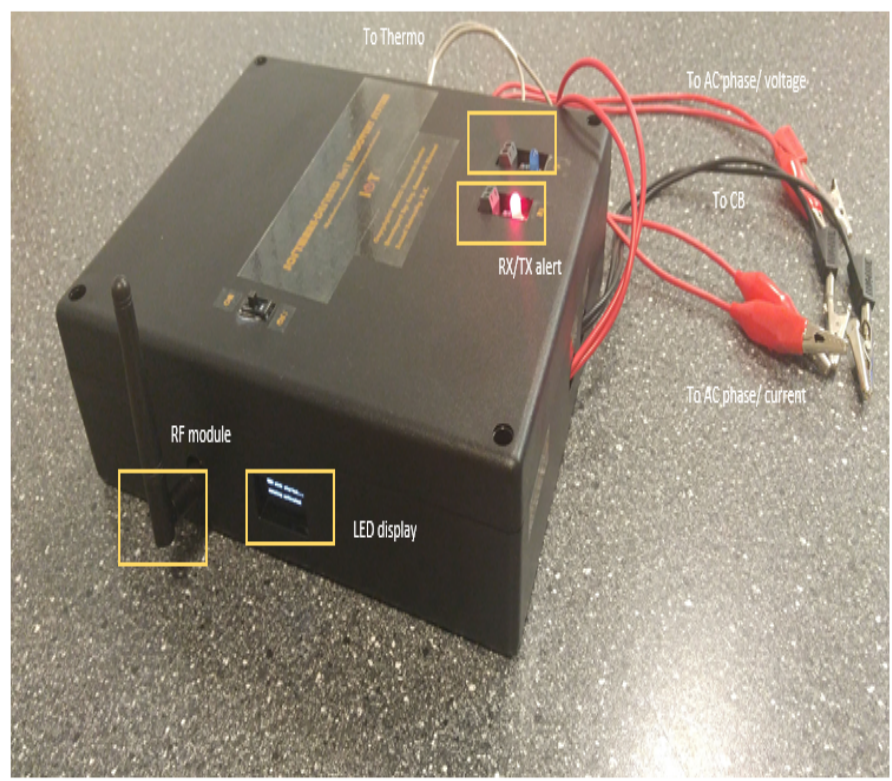

Fig. 7: finalized proposed sensor platform while in active mode
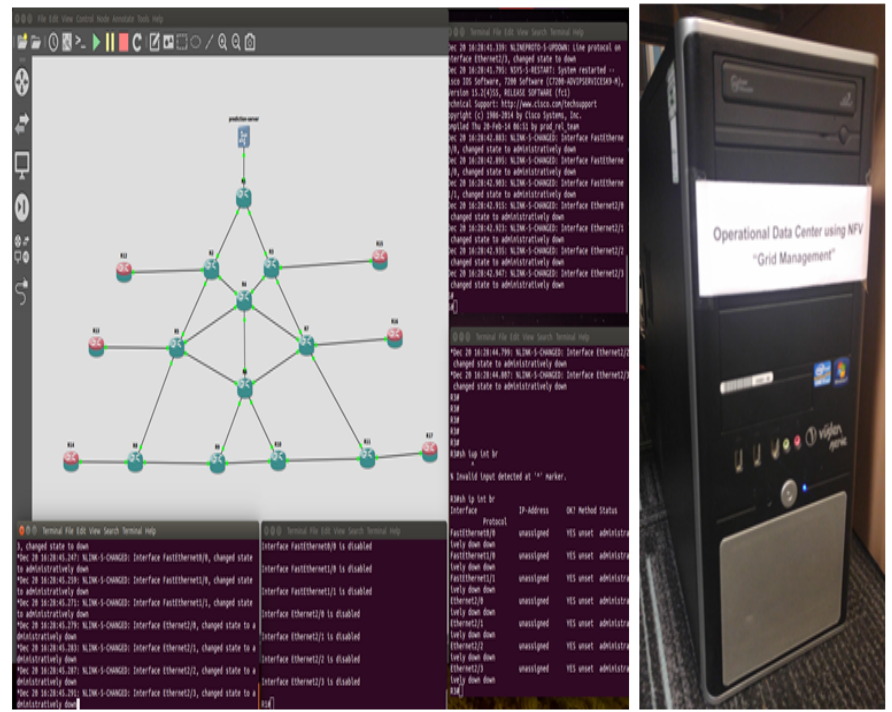

Fig. 8: proposed virtualized core topology implemented on a Linux server for sensor traffic management and fault prediction using python classification libraries.

the weights to minimize the cost function.

\section{CONCLUSION}

The current electrical grid system in Iraq need to be updated with new engineering implementation to overcome demand and outage challenges and adapt itself to new grid requirements to reduce maintenance cost. Therefore, this paper proposed a novel SDN IoT sensor platform to monitor the electrical parameters in distribution transformers to provide solutions for the electrical grid in Iraq. Current electrical grid weaknesses have been discussed and the effectiveness of our proposed system was highlighted along with the proposed prediction system. Experimental testing has been implemented 

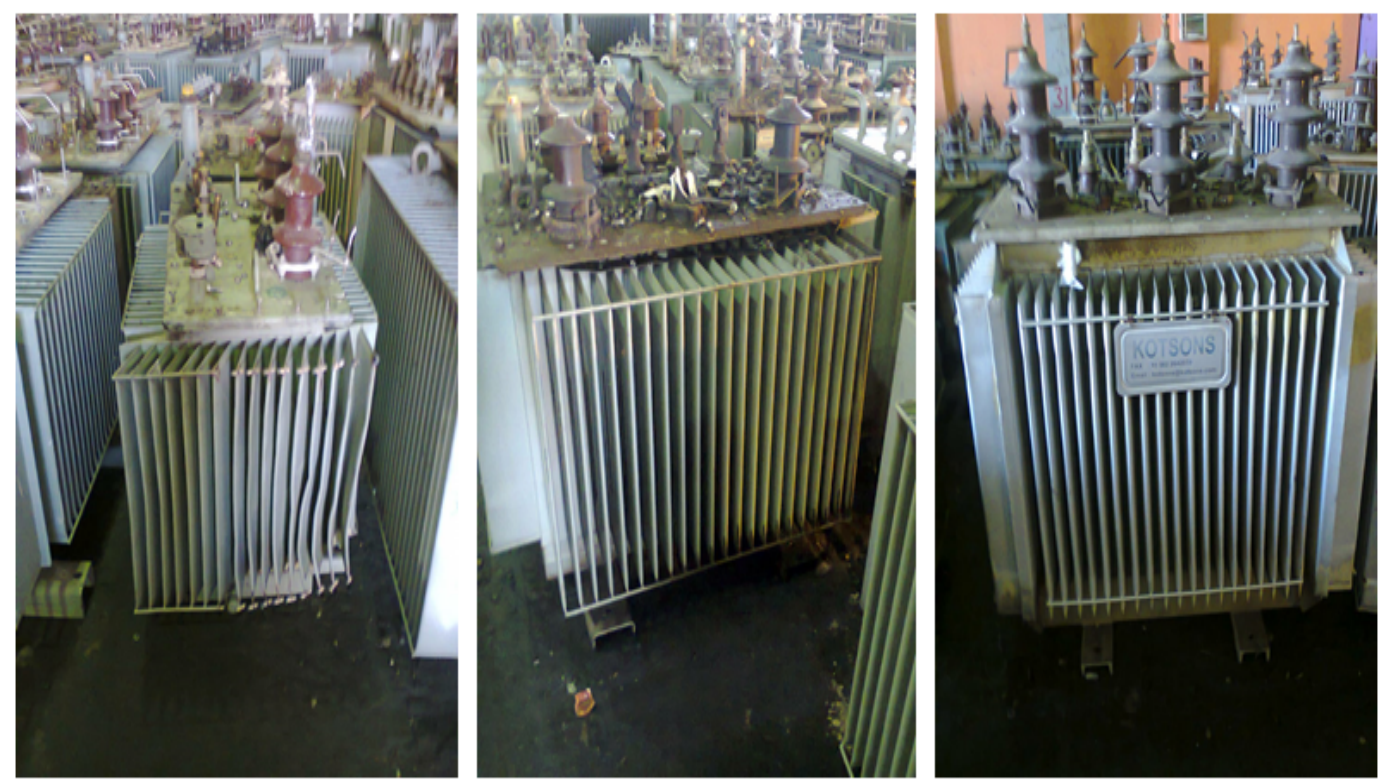

Fig. 9: Faulty DT samples from Baghdad electrical grid maintenance site as follows: pivot damage, oil leaks, and shorted winding.

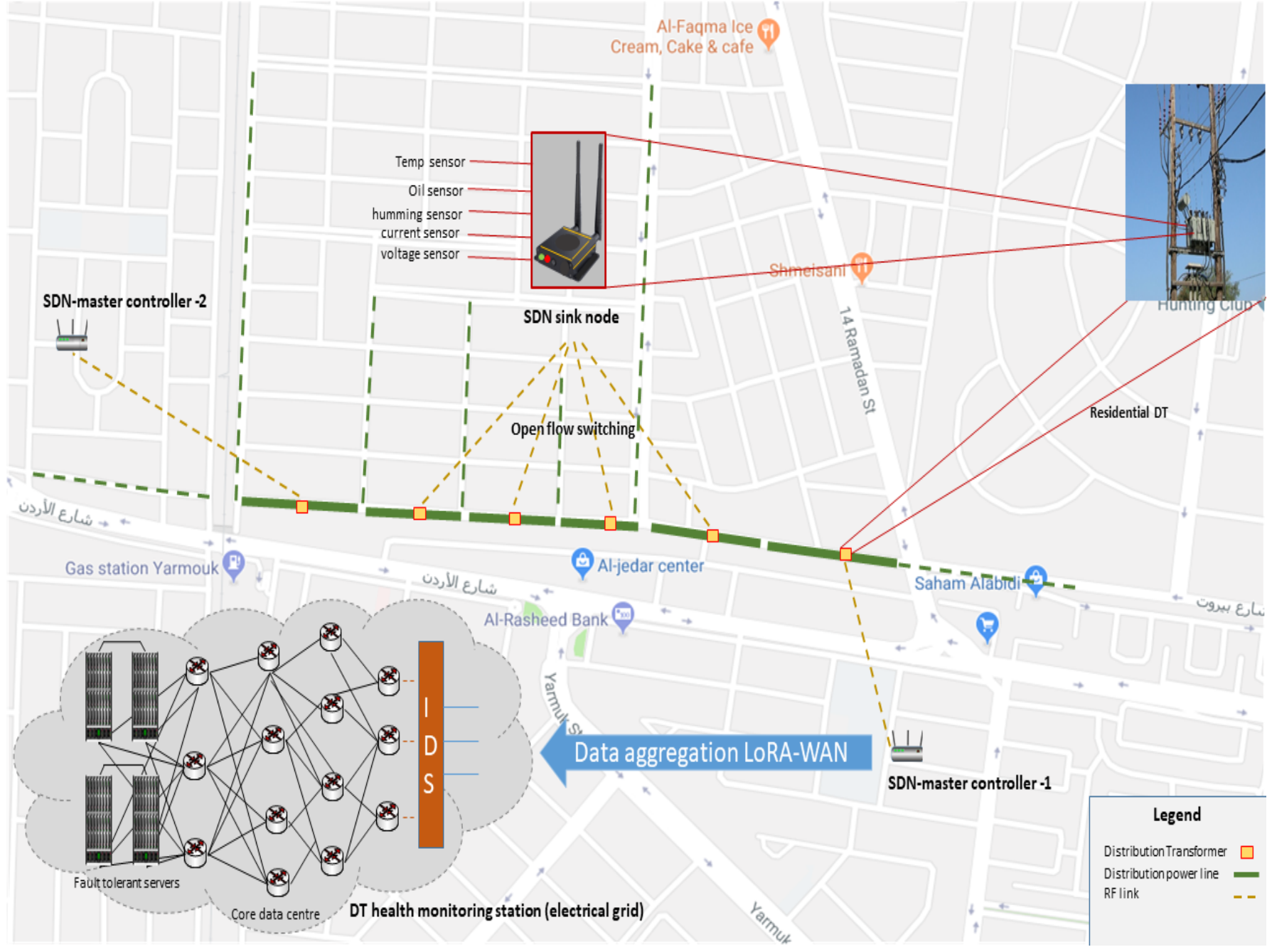

Fig. 10: proposed SDN sensor deployment scenario in a residential zone per DT platform 


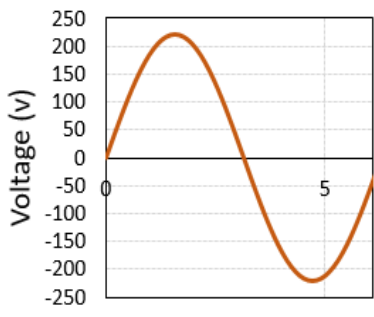

Time(ms)

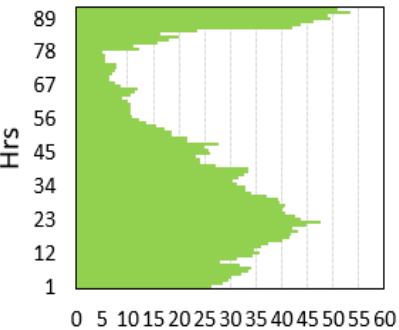

Noise $(\mathrm{db})$
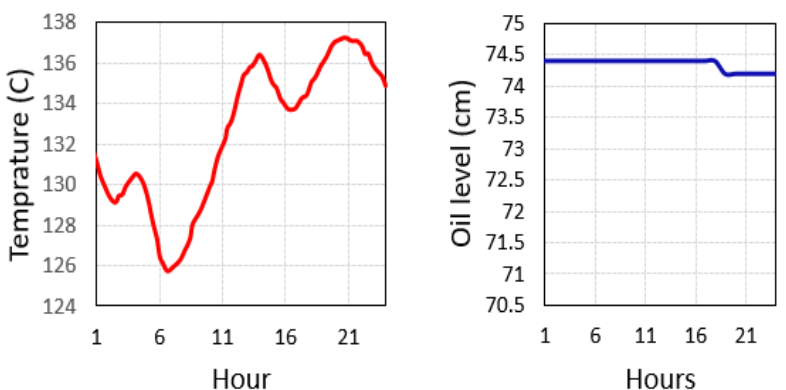

Fig. 11: sensor data collected for one phase from an operational miniature DT using our proposed testbed

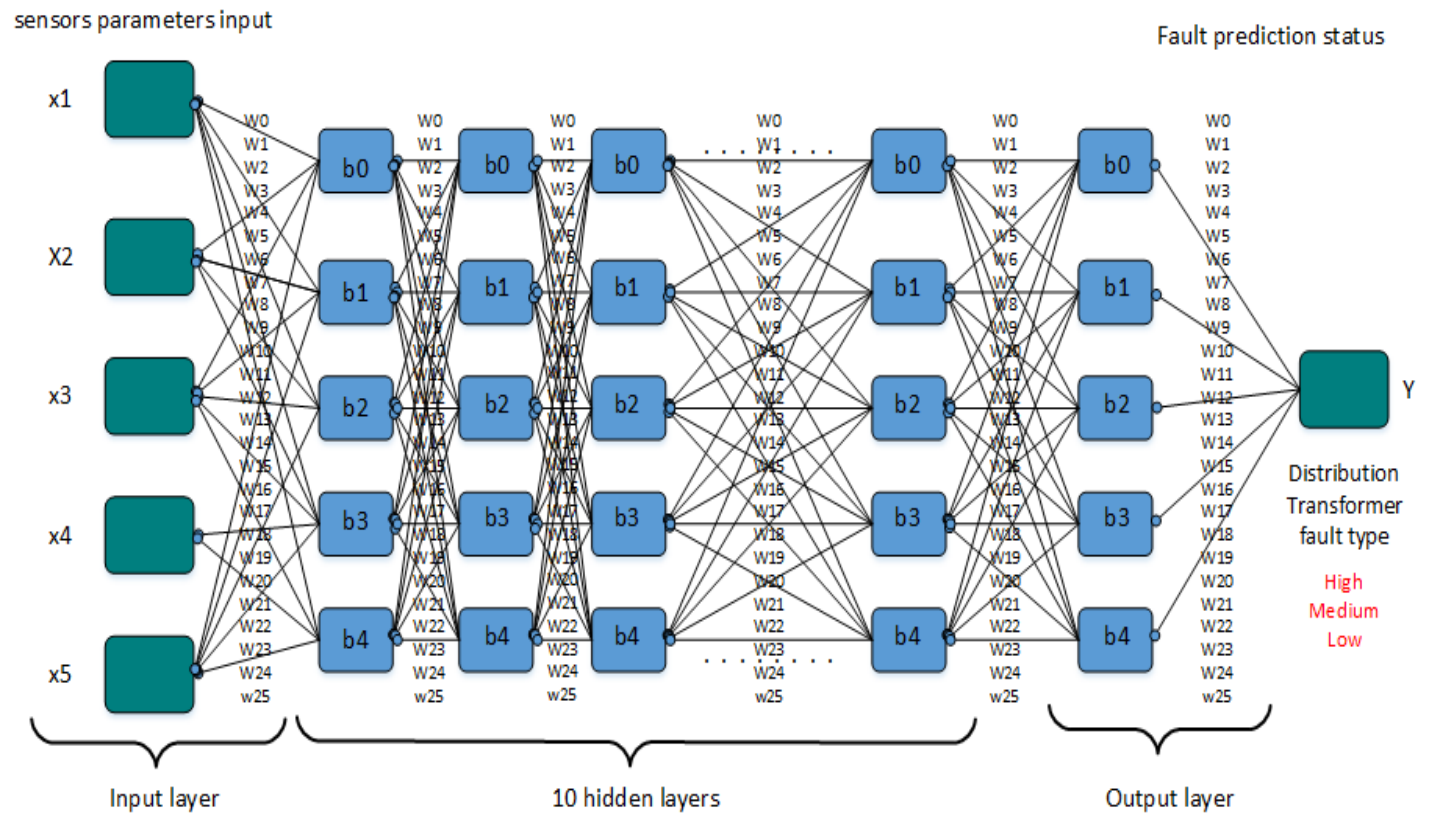

Fig. 12: proposed combined Feed-Forward and Decision Tree fault classification system structure

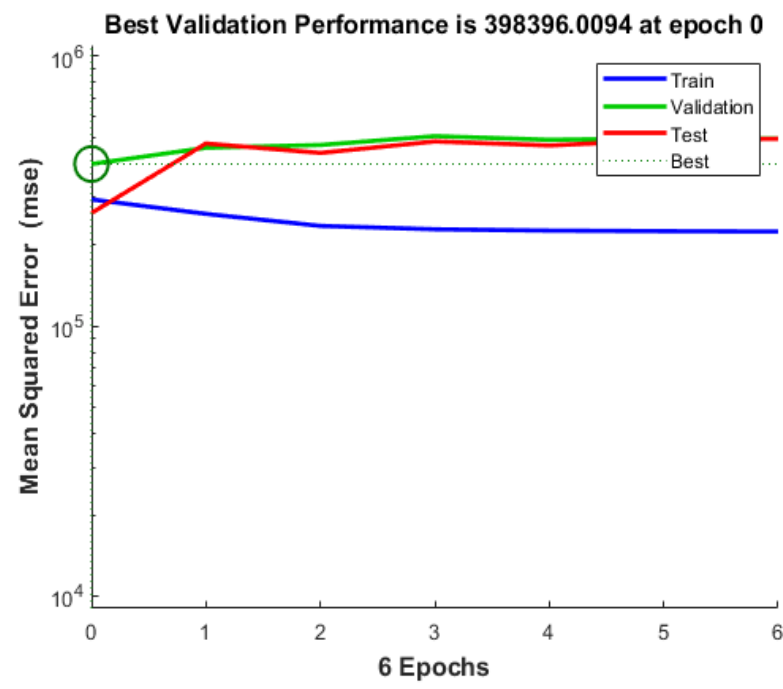

Fig. 13: objective function error rate

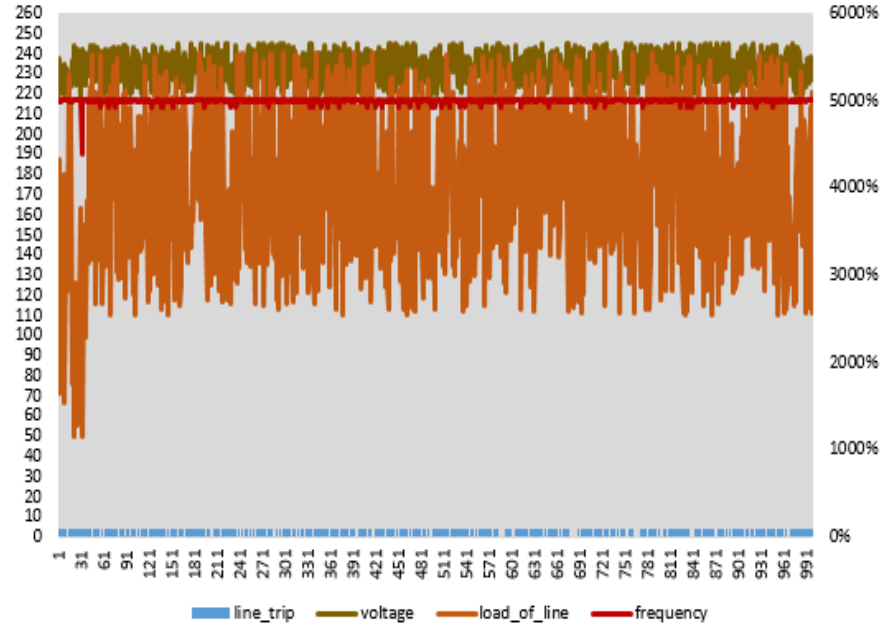

Fig. 14: data set used for training the network 

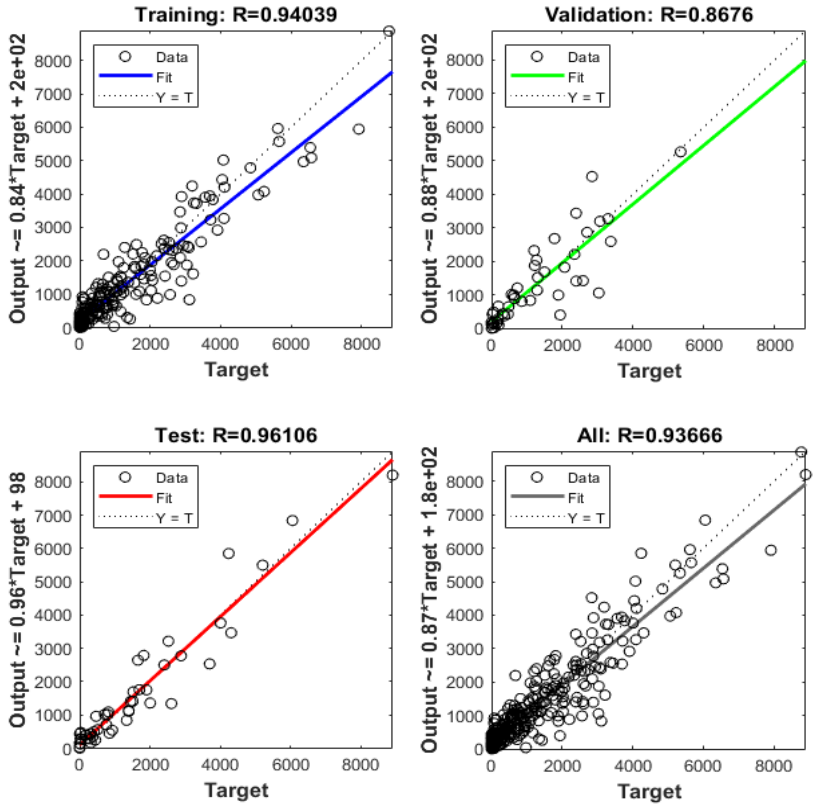

Fig. 15: prediction phases
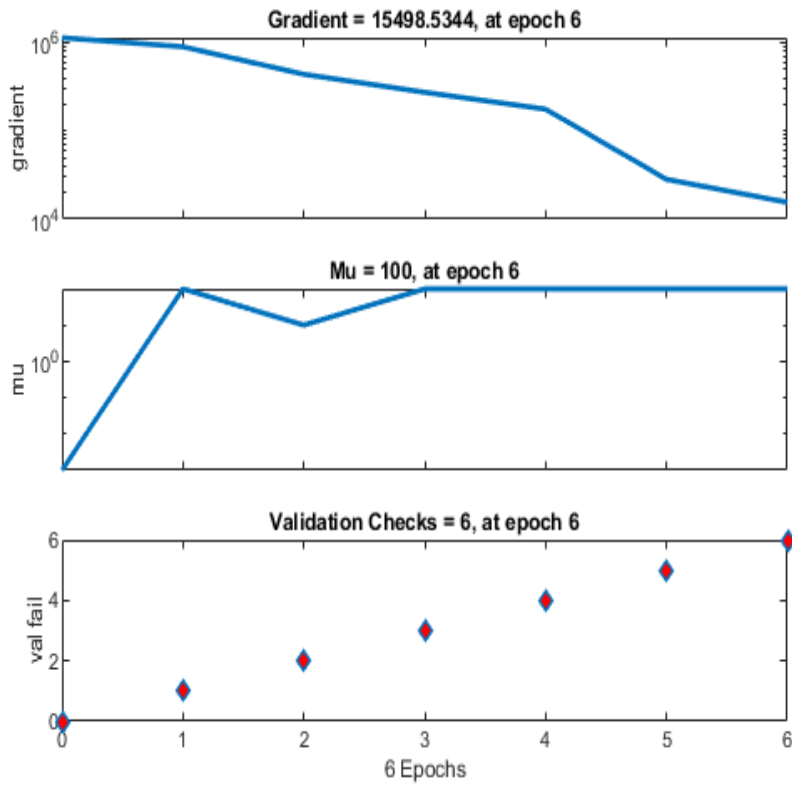

Fig. 16: gradient and validation checks

on an application case to validate the proposed prototype. The hardware was built using IoT hardware sensors and controllers. The controller was programmed as a customized SDN controller with the ability to operate as a sink and regular node. The testbed can also be connected to a circuit breaker to smartly manage any high alert threshold that cloud occur such as overload, high voltage, etc. The SDN-sense protocol was proposed to manage the communication of $N$ nodes efficiently. Moreover, we have implemented the data center on a virtual Linux server with multiple paths for redundancy. The prediction platform was implemented using a python library and Matlab simulation. Experimental results showed prediction results with $96.1 \%$ accuracy. In summary, the proposed system is considered to be low-cost implementation with realtime management that can provide a total overview of the DT status and to eliminate any future fails and outages that may occur in the distribution lines.

\section{REFERENCES}

[1] M. Wang, A. J. Vandermaar, and K. D. Srivastava, "Review of condition assessment of power transformers in service," IEEE Electrical insulation magazine, vol. 18, no. 6, pp. 12-25, 2002.

[2] K. Wang, J. Yu, Y. Yu, Y. Qian, D. Zeng, S. Guo, Y. Xiang, and J. Wu, "A survey on energy internet: Architecture, approach, and emerging technologies," IEEE Systems Journal, vol. 12, no. 3, pp. 2403-2416, 2018.

[3] S. Azodolmolky, Software Defined Networking with OpenFlow. packt publishing, 2003, vol. 21.

[4] E. C. Fontana, "Powering software defined networks with an any rack capable power bus," INTELEC, International Telecommunication Energy Conference (Proceedings), vol. 2016-Novem, pp. 1-4, 2016.

[5] T. D. Nadeau and K. Gray, SDN: Software Defined Networks: An Authoritative Review of Network Programmability Technologies. O'Reilly Media, Inc.”, 2013.

[6] S. Bailey, D. Bansal, L. Dunbar, D. Hood, Z. L. Kis, B. Mack-Crane, J. Maguire, D. Malek, D. Meyer, M. Paul, S. Schaller, F. Schneider, and R. Sherwood, "SDN Architecture Overview," Open Networking Foundation, 2013. [Online]. Available: https://www.opennetworking.org/images/stories/downloads/sdnresources/technical-reports/SDN-architecture-overview-1.0.pdf

[7] J. Li, D. Li, Y. Yu, Y. Huang, J. Zhu, and J. Geng, "Towards full virtualization of SDN infrastructure," Computer Networks, vol. 143, pp. 1-14, 2018. [Online]. Available: https://doi.org/10.1016/j.comnet.2018.06.014

[8] N. Bizanis and F. A. Kuipers, "SDN and Virtualization Solutions for the Internet of Things: A Survey," IEEE Access, vol. 4, pp. 5591-5606, 2016.

[9] IEC, "Internet of Things: Wireless Sensor Networks," IEC white paper. [Online]. Available: http://www.iec.ch

[10] I. N. da Silva, C. G. Gonzales, R. A. Flauzino, P. G. da Silva Junior, R. A. Fernandes, E. S. Neto, D. H. Spatti, and J. A. Ulson, "Intelligent systems for the detection of internal faults in power transmission transformers," in Advances in Expert Systems. InTech, 2012.

[11] E. Alpaydin, Introduction to machine learning. MIT press, 2014.

[12] R. Amin, M. Reisslein, and N. Shah, "Hybrid sdn networks: A survey of existing approaches," IEEE Communications Surveys \& Tutorials, 2018.

[13] S. Zhang, Y. Wang, M. Liu, and Z. Bao, "Data-based line trip fault prediction in power systems using $1 \mathrm{stm}$ networks and svm," IEEE Access, vol. 6, pp. 7675-7686, 2018.

[14] H. Ha and S. Subramanian, "Predicting the prospective fault level on distribution grids and its impact on protective relaying," in Protective Relay Engineers (CPRE), 2017 70th Annual Conference for. IEEE, 2017, pp. 1-4.

[15] Y. Di, C. Jin, B. Bagheri, Z. Shi, H. D. Ardakani, Z. Tang, and J. Lee, "Fault prediction of power electronics modules and systems under complex working conditions," Computers in Industry, vol. 97, pp. 1-9, 2018.

[16] M. Mahdi and V. I. Genc, "Artificial neural network based algorithm for early prediction of transient stability using wide area measurements," in Smart Grid and Cities Congress and Fair (ICSG), 2017 5th International Istanbul. IEEE, 2017, pp. 17-21.

[17] F. Xiao and Q. Ai, "Data-driven multi-hidden markov model-based power quality disturbance prediction that incorporates weather conditions," IEEE Transactions on Power Systems, 2018.

[18] Y. Kim, A. Goyal, and T. Kumar, "Predictive modeling of dissolved gas concentrationin oil-immersed substation transformers," in Smart Energy Grid Engineering (SEGE), 2016 IEEE. IEEE, 2016, pp. 261-267.

[19] H.-C. Lee and K.-H. Ke, "Monitoring of large-area iot sensors using a lora wireless mesh network system: Design and evaluation," IEEE Transactions on Instrumentation and Measurement, 2018

[20] Z. Lv, L. Wang, Z. Guan, J. Wu, X. Du, H. Zhao, and M. Guizani, "An optimizing and differentially private clustering algorithm for mixed data in sdn-based smart grid," IEEE Access, vol. 7, pp. 45 773-45 782, 2019. 
[21] K. Wang, Y. Wang, Y. Sun, S. Guo, and J. Wu, "Green industrial internet of things architecture: An energy-efficient perspective," IEEE Communications Magazine, vol. 54, no. 12, pp. 48-54, 2016.

[22] K. Wang, Y. Wang, D. Zeng, and S. Guo, "An sdn-based architecture for next-generation wireless networks," IEEE Wireless Communications, vol. 24, no. 1, pp. 25-31, 2017.

[23] K. Wang, Y. Shao, L. Shu, C. Zhu, and Y. Zhang, "Mobile big data fault-tolerant processing for ehealth networks," IEEE Network, vol. 30, no. 1 , pp. 36-42, 2016.

[24] M. Gao, K. Wang, and L. He, "Probabilistic model checking and scheduling implementation of an energy router system in energy internet for green cities," IEEE Transactions on Industrial Informatics, vol. 14, no. 4, pp. 1501-1510, 2018.

[25] Linux Foundation Projects, "OPNFV." [Online]. Available: https://www.opnfv.org/about

[26] Ubuntu 14.04.5 LTS (Trusty Tahr), 2018 (accessed October 8, 2014). [Online]. Available: http://releases.ubuntu.com/14.04/

[27] Mininet, 2018 (accessed October 8, 2014). [Online]. Available: http://mininet.org/

[28] Floodlight controller, 2018 (accessed October 8, 2014). [Online]. Available: http://www.projectfloodlight.org/floodlight/

[29] G. A. Valencia-Zapata, D. Mejia, G. Klimeck, M. Zentner, and O. Ersoy, "A statistical approach to increase classification accuracy in supervised learning algorithms," arXiv preprint arXiv:1709.01439, 2017.

[30] Arduino, "Ardunio board," 2018. [Online]. Available: https://www.arduino.cc/
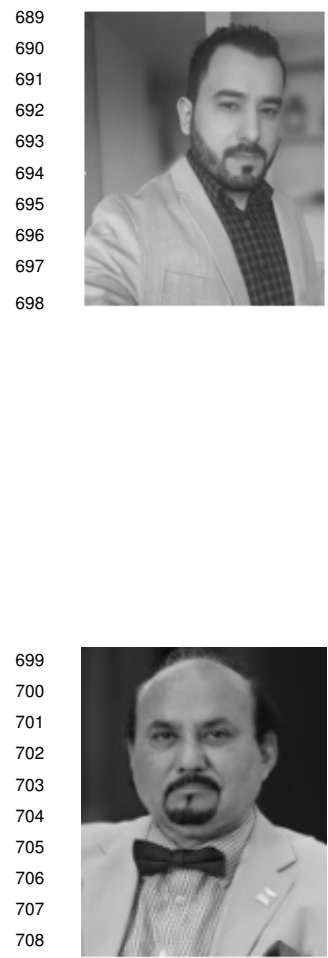

Hamed S. Al-Raweshidy received the B.Eng. and M.Sc. degrees from the University of Technology, Baghdad, Iraq, in 1977 and 1980, respectively, the Post Graduate Diploma degree from Glasgow University, Glasgow, U.K., in 1987, and the Ph.D. degree from the University of Strathclyde, Glasgow, in 1991. He was with: the Space and Astronomy Research Centre, Baghdad; PerkinElmer, Waltham, MA, USA; British Telecom, London, U.K.; Oxford University, Oxford, U.K.; Manchester Metropolitan University, Manchester, U.K.; and Kent University, Canterbury, U.K. He is currently the Director of the Wireless Network Communications Centre, Brunel University London. 